\title{
Autologous cell-free serum preparations in the management of knee osteoarthritis: what is the current clinical evidence?
}

\author{
Darshan S. Angadi , Hamish Macdonald and Navraj Atwal
}

\begin{abstract}
Background: There is paucity in the current literature regarding clinical outcomes of autologous cell-free serum preparations. The objective of this paper is to collate the clinical evidence and review the results of intraarticular injections of autologous cell-free serum preparations in the management of knee osteoarthritis (OA).

Methods: A comprehensive English literature search was undertaken using the healthcare database website (https://hdas.nice.org.uk/). The PubMed, Medline, CINAHL, Embase and the Cochrane library databases were searched to identify all studies of autologous protein solution/autologous conditioned serum (ACS/APS) in the management of knee OA. We evaluated the reported clinical outcomes with respect to pain, function, morbidity, adverse effects and complications.
\end{abstract}

Results: Fifteen relevant articles were identified in the current literature. Outcomes following injection of ACS/APS have been reported in patients with age range (34-87 years) and unilateral or bilateral knee OA. Seven studies reported improvement in visual analog scale (VAS) whereas the Western Ontario and McMaster Universities osteoarthritis instrument (WOMAC) score improved in nine studies. Considerable variation was noted in the injection technique and duration of post-procedure assessment with only one study reporting long-term follow-up beyond 24 months. Joint swelling and injection-site pain were reported to be the most common complications; only one study reported a case of septic arthritis. However, no evidence is available to clearly identify factors that may predict the outcomes following this procedure.

Conclusion: Current data from the clinical studies would suggest that the intraarticular administration of autologous cell-free serum preparations, such as ACS/APS, in patients with knee OA may improve pain and function, with limited morbidity. High-quality clinical trials with stratified patient cohorts, longer follow-up duration and robust reporting of outcome measures are essential to improve the understanding of the indications and clinical effectiveness of these novel products.

Keywords: Autologous, Protein, Solution, Conditioned, Serum, Osteoarthritis, Knee

\footnotetext{
* Correspondence: docdarshan@gmail.com

Department of Trauma and Orthopaedics, Cheltenham General Hospital,

Sandford Rd, Cheltenham, UK

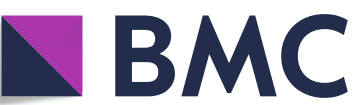

Part of Springer Nature

(c) The Author(s). 2020 Open Access This article is licensed under a Creative Commons Attribution 4.0 International License, which permits use, sharing, adaptation, distribution and reproduction in any medium or format, as long as you give appropriate credit to the original author(s) and the source, provide a link to the Creative Commons licence, and indicate if changes were made. The images or other third party material in this article are included in the article's Creative Commons licence, unless indicated otherwise in a credit line to the material. If material is not included in the article's Creative Commons licence and your intended use is not permitted by statutory regulation or exceeds the permitted use, you will need to obtain permission directly from the copyright holder. To view a copy of this licence, visit http://creativecommons.org/licenses/by/4.0/. The Creative Commons Public Domain Dedication waiver (http://creativecommons.org/publicdomain/zero/1.0/) applies to the data made available in this article, unless otherwise stated in a credit line to the data.
} 


\section{Background}

Osteoarthritis (OA) of the knee is common [1], debilitating [2] and increasing in its prevalence [1]. Any therapeutic intervention that can relieve symptoms of OA, prevent its progression and/or delay the need for surgery therefore has potential to dramatically improve quality of life for patients. Nonoperative treatment modalities for symptomatic knee OA include analgesia [3], physiotherapy [4], healthy lifestyle and weight reduction regimens [5]. Surgical intervention in the form of osteotomy [6] or arthroplasty [7] is well-established, but is not without complications [8] and a prolonged rehabilitation phase [9].

Intraarticular therapies for knee OA using intraarticular corticosteroid (IAC) and hyaluronic acid (HA) have been reported [10]. However, dose-dependent chondrotoxicity [11], short duration of effect [12] with IAC and arthralgia [13], and variable evidence [14] with HA are some of their limitations. Some investigators have performed intraarticular injection of platelet-rich plasma (PRP) [15] and mesenchymal stem/stromal cells (MSCs) [16] due to their perceived biological function(s) to restore joint homeostasis $[12,17]$. The pathophysiology of OA includes a complex interplay of pro-inflammatory mediators like interleukin-1 (IL-1), interleukin-6 (IL-6), tumor necrosis factor-alpha (TNF-a), macrophage chemotactic protein (MCP), monokine induced by interferon (MIG), oncostatin M (OSM) and matrix metalloproteinases (MMPs) amongst others. The biological therapeutic group products which have been developed for the management of OA of the knee are designed to inhibit the action of interleukin-1 (IL-1), a pro-inflammatory cytokine that has been implicated in, and targeted for treatment of, multiple human diseases [12, 17, 18]. Animal experimental evidence suggests that upregulation of IL-1-receptor antagonist (IL-1Ra) may reduce the progression of OA [19]. Biological injective therapy options based around this theory have been developed and referred to as autologous conditioned serum (ACS) or autologous protein solution (APS), both of which are prepared from autologous peripheral blood, which is conditioned by incubation with glass beads, leading to increases in the production of IL-1Ra as well as multiple other cytokines and growth factors [17].

Several investigators have evaluated the role of autologous cell-free serum preparations like APS [20-22] and ACS [23-25] in the management of knee OA with human clinical studies following initial in-vitro $[18,26]$ and animal model studies $[19,27]$. The majority of the available review articles on the topic have summarised the basic science and the results of ACS. However, there is paucity in the current literature providing an objective evaluation of the clinical outcomes of these novel products.
Hence, the primary objective of this paper was to collate the available clinical evidence in the published literature and critically appraise the results of intraarticular injection of autologous cell-free serum preparations in the management of knee OA. The secondary objective was to answer the following questions encountered in the clinical decision-making process of managing patients with OA of the knee:

1. Is it safe to perform intraarticular injection of autologous cell-free serum preparations such as APS/ACS for OA of the knee using commercially available kits?

2. Does this procedure provide an effective and longlasting symptomatic relief to avoid further surgical intervention?

3. What factors (patient/procedure/device) significantly influence the outcome following this treatment?

4. Do these intraarticular injection procedures have any potential adverse effect on the outcomes of subsequent surgical procedures?

Given the similarities, we included studies pertaining to both, with the generic title of ACS/APS referring to both products.

\section{Methods}

\section{Literature search and databases}

An English literature search of all the available evidence was undertaken (June 2019) using the healthcare database website (https://hdas.nice.org.uk/). The databases searched were Medline, CINAHL, Embase and the Cochrane library.

\section{Search criteria}

Medline search was performed using Boolean statements and the wildcard symbol (*). The search criteria: "knee* AND (auto* OR autologous*) AND (condition* OR conditioned* OR protein* OR pro*) AND (serum* OR solution* OR solutions")". Embase search was performed using Boolean statements and the wildcard symbol $(*)$. The search criteria: "knee* AND (auto* OR autologous*) AND (condition* OR conditioned* OR protein* OR pro*) AND (serum* OR solution* OR solutions")". CINAHL database was searched using the following criteria: "knee" AND (auto" OR autologous*) AND (condition* OR conditioned" OR protein* OR pro*) AND (serum* OR solution* OR solutions")".

A review of the Cochrane database for relevant articles was performed. An adjunctive bibliography search was undertaken to identify additional relevant studies through review articles and Google scholar (https:// scholar.google.co.uk/) using the commercial names of 
APS -'nSTRIDE'(Biomet Biologics, Warsaw, IN, USA) and ACS -'Orthokin'(Orthogen AG, Dusseldorf, Germany). Additionally, given the scope of the current review to assess the safety of this novel treatment including adverse reactions, a comprehensive search of the grey literature (OpenGrey [28]/OpenDOAR [29]) was undertaken. All studies reporting the clinical outcomes of patients with knee OA receiving intraarticular injection of autologous cell-free preparations like APS/ACS were included. Review articles, animal and in-vitro studies were excluded.

\section{Results}

The above database search returned 555 articles of which 27 were relevant to the current review. Adjunctive bibliography search identified three articles whereas 15 articles were noted to be duplicates (Table 1). Grey literature search found no results. Thus, a total of 15 relevant articles were identified in the current literature and were selected for further review.

It was noted that amongst the 15 articles, two were initial abstracts $[25,30]$ of subsequent papers in the same cohort of patients. Hence, data from the later studies was considered for analysis. Details of the articles which describe the clinical outcomes of intraarticular injection of autologous cell-free serum preparations like APS/ACS are provided in Tables 2, 3, 4 and 5. The salient points and current evidence to the focussed questions to help in the clinical decision-making process are presented below.

\section{Safety profile and morbidity of intraarticular injections of ACS/APS}

To date, the outcomes of 1229 patients involved in studies evaluating the effect of injection of ACS/APS have been reported (Table 2). The adverse reactions noted in these patients have been similar to the intraarticular administration of other therapeutic agents [17, 40]. Additionally, they have been of a transient and self-limiting type [12, 41]. Following clinical trials, the investigators from the above studies in the current literature have concluded that the overall safety profile of autologous cell-free serum preparations, such as ACS and APS, to be satisfactory for clinical use. Ongoing clinical trials [42] have the scope to provide further evidence in this aspect. Amongst the various studies in the literature only one study from 2008 reported a single case of septic arthritis [39]. However, this complication has not been reported by other investigators.

\section{Duration of symptom relief following ACS/APS injection}

Current studies have demonstrated that duration of pain and symptom relief period can vary from 3 to 24 months (Tables 2 and 4). Zarringam et al. [23] performed Kaplan-Meier survivorship analysis on the original cohort of patients from the study conducted by Yang et al. [39] between February 2004 to August 2006. They noted that at $7.5 \pm 3.9$ years of follow-up, $40.3 \%$ of patients from the group who received ACS compared to $46.3 \%$ of patients from the placebo group who received physiological saline underwent surgical intervention. However, this was not statistically significant $(p=0.150)$.

\section{Factors influencing the outcome of ACS/APS treatment Patient demographics and physiology}

Outcomes following injection of ACS/APS have been reported in patients over a wide age range (34-87 years) with unilateral or bilateral knee OA. All the investigational studies of these products have been performed on cohorts of patients with symptomatic knee OA excluding patients with conditions like systemic/inflammatory joint disease and crystalline/ neuropathic arthropathy. It must be noted that whilst some investigators excluded patients who had had surgery on their knee within 3 months [31, 38] of the screening visit others have extended this time limit to 6 [22] and 12 months [21]. Furthermore, there is significant variation in the inclusion criteria with respect to patients who have received intraarticular treatments. Body mass index (BMI) of the study patients is not consistently reported in the literature (Table 2).

\section{Indications and severity of $O A$}

ACS/APS injections have been performed in patients with symptomatic unilateral or bilateral knee OA with

Table 1 Results of literature search

\begin{tabular}{lccccc}
\hline & Database & & & Total \\
\cline { 2 - 5 } & Medline & Embase & CINAHL & Cochrane \\
\hline Search results & 132 & 362 & 50 & 11 & 355 \\
Relevant articles & 8 & 11 & 5 & 27 \\
Adjunctive bibliography & & & & 03 \\
Duplicates & & & & 15 \\
Studies for review & & & 15 \\
\hline
\end{tabular}


Table 2 List of relevant studies from literature

\begin{tabular}{|c|c|c|c|c|c|c|c|c|c|}
\hline $\begin{array}{l}\text { Author and } \\
\text { year }\end{array}$ & $\begin{array}{l}\text { Study } \\
\text { patients }\end{array}$ & $\begin{array}{l}\text { Age at } \\
\text { presentation } \\
\text { Mean }( \pm \\
\text { SD)/median } \\
\text { (range) }\end{array}$ & $\begin{array}{l}\text { BMl } \\
\text { Mean }( \pm \\
\text { SD)/median } \\
\text { (range) }\end{array}$ & $\begin{array}{l}\text { OA severity (K-L } \\
\text { grade/ACR } \\
\text { criteria) }\end{array}$ & $\begin{array}{l}\text { Intraarticular } \\
\text { injection and } \\
\text { number }\end{array}$ & $\begin{array}{l}\text { Follow-up (months) } \\
\text { Mean ( } \pm \text { SD)/ } \\
\text { median (range) }\end{array}$ & $\begin{array}{l}\text { Outcome } \\
\text { measures }\end{array}$ & $\begin{array}{l}\text { Adverse events } \\
\text { (AE)/ } \\
\text { complications }\end{array}$ & $\begin{array}{l}\text { Level of } \\
\text { evidence }\end{array}$ \\
\hline $\begin{array}{l}\text { Kon et al. } \\
2018 \text { [22] }\end{array}$ & 46 & $57(41-68)$ & NR & K-L 2-3 & $\begin{array}{l}\text { APS } \\
1\end{array}$ & $12( \pm N R)$ & $\begin{array}{l}\text { WOMAC } \\
\text { VAS } \\
\text { KOOS } \\
\text { SF-36 } \\
\text { CGI-S/ } \\
\text { PGI-S }\end{array}$ & $\begin{array}{l}\mathrm{AE}-6 \\
\text { Arthralgia }\end{array}$ & 2 \\
\hline $\begin{array}{l}\text { Tassara et al. } \\
2018 \text { [24] }\end{array}$ & 25 & $68(34-87)$ & NR & NR & $\begin{array}{l}\text { ACS } \\
4\end{array}$ & 6 ( $\pm N R)$ & $\begin{array}{l}\text { VAS } \\
\text { ROM }\end{array}$ & None & 3 \\
\hline $\begin{array}{l}\text { Zarringam } \\
\text { et al. } 2018 \text { [23] }\end{array}$ & 126 & 63 (NR) & NR & K-L 1-3 & $\begin{array}{l}\text { ACS } \\
6\end{array}$ & $90( \pm 47)$ & K-M & $N R$ & 3 \\
\hline $\begin{array}{l}\text { Barreto et al. } \\
2017 \text { [31, 32] }\end{array}$ & 100 & $61.2( \pm 1.2)$ & $33.8( \pm 1.4)$ & $A C R$ & $\begin{array}{l}\text { ACS } \\
6\end{array}$ & $12( \pm N R)$ & $\begin{array}{l}\text { VAS } \\
\text { XSMFA-D } \\
\text { PGIC }\end{array}$ & $N R$ & 3 \\
\hline $\begin{array}{l}\text { Hang et al. } \\
2017 \text { [33] }\end{array}$ & 92 & NR & NR & NR & $\begin{array}{l}\text { ACS } \\
4\end{array}$ & $12( \pm N R)$ & $\begin{array}{l}\text { VAS } \\
\text { WOMAC }\end{array}$ & $N R$ & 3 \\
\hline $\begin{array}{l}\text { Shirokova } \\
\text { et al. } 2017 \text { [34] }\end{array}$ & 123 & $59.9( \pm 8.8)$ & NR & $A C R$ & $\begin{array}{l}\text { ACS } \\
6\end{array}$ & $3( \pm N R)$ & $\begin{array}{l}\text { VAS } \\
\text { WOMAC }\end{array}$ & $N R$ & 3 \\
\hline $\begin{array}{l}\text { Hix et al. } \\
2017 \text { [20] }\end{array}$ & 10 & $58.8( \pm 9.5)$ & $29.0( \pm 3.9)$ & NR & $\begin{array}{l}\text { APS } \\
1\end{array}$ & $12( \pm N R)$ & $\begin{array}{l}\text { WOMAC } \\
\text { KOOS } \\
\text { NRS }\end{array}$ & $\begin{array}{l}\text { AE - 4 } \\
\text { Arthralgia } \\
\text { Discomfort }\end{array}$ & 3 \\
\hline $\begin{array}{l}\text { van Drumpt } \\
\text { et al. } 2016 \text { [21] }\end{array}$ & 10 & $57.5( \pm 9.5)$ & $26.6( \pm 3.1)$ & K-L 1-4 & $\begin{array}{l}\text { APS } \\
1\end{array}$ & $18( \pm 1)$ & WOMAC & $\begin{array}{l}\text { AE - } 6 \\
\text { Arthralgia } \\
\text { Joint stiffness } \\
\text { Injection site } \\
\text { pain }\end{array}$ & 3 \\
\hline $\begin{array}{l}\text { Garcia- } \\
\text { Escudero et al. } \\
2015 \text { [35] }\end{array}$ & 118 & $59(34-81)$ & 29.6 ( $\pm N R)$ & K-L 1-4 & $\begin{array}{l}\text { ACS } \\
4\end{array}$ & $24( \pm N R)$ & $\begin{array}{l}\text { WOMAC } \\
\text { NRS }\end{array}$ & None & 3 \\
\hline $\begin{array}{l}\text { Rutgers et al. } \\
2015 \text { [36] }\end{array}$ & 20 & $50(34-70)$ & NR & K-L 1-3 & $\begin{array}{l}\text { ACS } \\
6\end{array}$ & $12( \pm N R)$ & $\begin{array}{l}\text { VAS } \\
\text { KOOS } \\
\text { KSCRS }\end{array}$ & $N R$ & 3 \\
\hline $\begin{array}{l}\text { Motaal et al. } \\
2014 \text { [37] }\end{array}$ & 30 & $\begin{array}{l}54.21( \pm \\
5.95)\end{array}$ & $N R$ & K-L 1-3 & $\begin{array}{l}\text { ACS } \\
3\end{array}$ & $3( \pm N R)$ & WOMAC & $N R$ & 3 \\
\hline $\begin{array}{l}\text { Baltzer et al. } \\
2009 \text { [38] }\end{array}$ & 376 & $53.8( \pm 12.2)$ & $N R$ & K-L 2-3 & $\begin{array}{l}\text { ACS } \\
6\end{array}$ & 25 ( $\pm N R)$ & $\begin{array}{l}\text { WOMAC } \\
\text { SF-8 } \\
\text { VAS }\end{array}$ & $\begin{array}{l}\text { AE - } 31 \\
\text { Joint swelling } \\
\text { Transient pain }\end{array}$ & 2 \\
\hline $\begin{array}{l}\text { Yang et al. } \\
2008 \text { [39] }\end{array}$ & 153 & $54( \pm 11)$ & $27( \pm 5)$ & K-L 1-3 & $\begin{array}{l}\text { ACS } \\
6\end{array}$ & $12( \pm N R)$ & $\begin{array}{l}\text { WOMAC } \\
\text { KSCRS }\end{array}$ & $\begin{array}{l}\text { Knee pain }-44 \\
\text { Irritation }-70 \\
\text { Swelling }-10 \\
\text { Septic arthritis } \\
-1\end{array}$ & 2 \\
\hline
\end{tabular}

$A E$ adverse events, $B M I$ body mass index, ROM range of movement, $S D$ standard deviation, NR not reported, APS autologous protein solution, $A C S$ autologous conditioned serum, K-L Kellgren-Lawrence, ACR American College of Rheumatology criteria, VAS visual analogue scale, KOOS Knee injury and Osteoarthritis Outcome Score, WOMAC Western Ontario and McMaster Universities osteoarthritis instrument, OA osteoarthritis, SF-8 Short-Form 8 health-related quality of life, $K$ M Kaplan-Meier method, CGI-S Clinical Global Impression of Severity, PGI-S Patient Global Impression of Severity, XSMFA-D Extra Short Musculoskeletal Functional Assessment, KSCRS Knee Society clinical rating scale, NR

radiographic changes (Kellgren-Lawrence grade 1 to 4 ). However, there is no clear agreement in terms of the objective criteria used to assess the severity of knee OA prior to performing these injections in the study patients. A combination of American College of Rheumatology (ACR) criteria [31, 38], visual analogue scale (VAS) [38, 39], numeric rating scale (NRS) [35], Western Ontario and McMaster Universities osteoarthritis instrument (WOMAC) [21, 22, 39], including the pain subscale, have been used by the investigators.

\section{Mechanical parameters (range of motion/lower limb alignment)}

Only one study [24] in the literature has evaluated the effect of the intraarticular administration of ACS on range of motion. Tassara et al. [24] noted that the 
Table 3 Reported outcomes - visual analogue scale (VAS) following intraarticular injection of autologous conditioned serum/ autologous protein solution (ACS/APS)

\begin{tabular}{|c|c|c|c|c|c|c|c|}
\hline \multirow[t]{2}{*}{ Author and year } & \multirow{2}{*}{$\begin{array}{l}\text { Study } \\
\text { patients }\end{array}$} & \multirow{2}{*}{$\begin{array}{l}\text { Age at presentation } \\
\text { Mean } \\
( \pm \text { SD)/median (range) }\end{array}$} & \multirow{2}{*}{$\begin{array}{l}\text { Follow-up (months) } \\
\text { Mean } \\
( \pm \text { SD)/median (range) }\end{array}$} & \multirow{2}{*}{$\begin{array}{l}\text { Outcome } \\
\text { measure }\end{array}$} & \multirow{2}{*}{$\begin{array}{l}\text { Baseline } \\
\text { Score } \\
\text { Mean }( \pm S D) / \text { median } \\
\text { (range) }\end{array}$} & \multicolumn{2}{|c|}{ Post procedure } \\
\hline & & & & & & $\begin{array}{l}\text { Time } \\
\text { point(s) } \\
\text { (weeks/ } \\
\text { months) }\end{array}$ & $\begin{array}{l}\text { Score } \\
\text { Mean }( \pm \text { SD)/ } \\
\text { median (range) }\end{array}$ \\
\hline Kon et al. 2018 [22] & 46 & $57(41-68)$ & 12 ( \pm NR) & VAS & 5.5 ( $\pm \mathrm{NR})$ & 12 months & $N R^{a}$ \\
\hline \multirow{2}{*}{$\begin{array}{l}\text { Tassara } \\
\text { et al. } 2018 \text { [24] }\end{array}$} & \multirow[t]{2}{*}{25} & \multirow[t]{2}{*}{$68(34-87)$} & \multirow[t]{2}{*}{$6( \pm N R)$} & \multirow[t]{2}{*}{ VAS } & \multirow[t]{2}{*}{$80(70-100)$} & 1 month & $20(0-60)$ \\
\hline & & & & & & 6 months & $20(0-60)$ \\
\hline \multirow{5}{*}{$\begin{array}{l}\text { Barreto } \\
\text { et al. } 2017[31,32]\end{array}$} & \multirow[t]{5}{*}{100} & \multirow[t]{5}{*}{$61.2( \pm 1.2)$} & \multirow[t]{5}{*}{12 ( $\pm \mathrm{NR})$} & \multirow[t]{5}{*}{ VAS } & \multirow[t]{5}{*}{$5.8( \pm 0.6)$} & 1 week & $4.2( \pm N R)$ \\
\hline & & & & & & 2 weeks & $3.3( \pm N R)$ \\
\hline & & & & & & 3 months & $3.1( \pm N R)$ \\
\hline & & & & & & 6 months & $3.2( \pm N R)$ \\
\hline & & & & & & 12 months & $2.3( \pm N R)$ \\
\hline Hang et al. 2017 & 92 & $N R$ & $12( \pm \mathrm{NR})$ & VAS & $6.07( \pm N R)$ & 12 months & $2.52( \pm N R)$ \\
\hline $\begin{array}{l}\text { Shirokova et al. } \\
2017 \text { [34] }\end{array}$ & 123 & $59.9( \pm 8.8)$ & $3( \pm N R)$ & VAS & $N R^{b}$ & 3 months & $N R^{b}$ \\
\hline $\begin{array}{l}\text { Rutgers } \\
\text { et al. } 2015 \text { [36] }\end{array}$ & 20 & $50(34-70)$ & $12( \pm N R)$ & VAS & $52.20( \pm 22.90)$ & 12 months & $50.05( \pm 23.76)$ \\
\hline $\begin{array}{l}\text { Baltzer } \\
\text { et al. } 2009 \text { [38] }\end{array}$ & 376 & $53.8( \pm 12.2)$ & $25( \pm N R)$ & VAS & $69.6( \pm 13.10)$ & 6 months & $29.5( \pm 22.58)$ \\
\hline
\end{tabular}

$N R$

a visual analogue scale (VAS) at 12 months reported to be $49 \%$ better but numeric score not provided

${ }^{b}$ VAS at 3 months reported to be $47 \%$ better but VAS value not provided

median range of knee flexion improved by $25^{\circ}$ to $120^{\circ}$ $\left(95-140^{\circ}\right)$ from a baseline of $95^{\circ}\left(90-105^{\circ}\right)$. Currently, there is no data to draw conclusions regarding varus/valgus alignment of the knee and its influence on the outcome following injection of ACS/APS.

\section{Injection technique and regimen}

Considerable variation is noted with the injection regimen for ACS (Table 2). Some investigators [31, 37] have used $1 \mathrm{ml}$ whilst others [24, 34, 35, 39] have administered $2 \mathrm{ml}$ of ACS. Furthermore, the number of injections performed is variable ranging between three [37] to six $[31,36]$ over a period of $2-3$ weeks. In contrast all the studies [20-22] with APS used a single injection of $2.5 \mathrm{ml}$. Some authors [21, 23, 39] recommend aspiration of knee prior to injection whilst others have used ultrasound-guided injections [22].

\section{Implications for future surgical procedures}

Five studies [21, 23, 35, 36, 38] have described further procedures carried out on participants subsequent to intraarticular treatment with ACS/APS. Only limited and indirect data is available from the current studies regarding patients who have undergone surgical procedures such as partial/total knee replacement [23, 35] and osteotomy [23]. In patients who had previously received ACS/APS there were a total of 35 additional procedures (26 arthroplasties, four osteotomies, two arthroscopic interventions and three unknown surgeries). To date no adverse outcome has been reported in this sub-group of patients following surgical intervention. One study [38] stated that 122 patients received further interventions, including surgical, pharmacological and complementary therapies, but did not give further information or stratify these by the therapy previously provided (ACS, HA or saline placebo). None of the trials specifically examined complications following subsequent procedures.

\section{Discussion}

Basic science of ACS/APS

Production methods

Currently, several commercially available kits are utilised to prepare ACS/APS from whole-blood samples of patients $[32,38,43]$. In general, both ACS and APS are prepared from autologous peripheral blood, the volume of which can vary between 10 and $60 \mathrm{ml}$ depending on the manufacturer kit [20, 37, 38]. The obtained blood sample is further conditioned by incubation with glass beads and centrifugation, leading to an increase in the production of IL-1Ra as well as multiple other cytokines and growth factors $[17,20]$.

\section{Composition}

The predominant cytokine in ACS is IL-1Ra with an average concentration of 2015 compared to 236 picograms per milliliter $(\mathrm{pg} / \mathrm{ml})$ in a basal blood sample [41]. Additional 
Table 4 Reported outcomes - Western Ontario and McMaster Universities osteoarthritis instrument (WOMAC) following intraarticular injection of autologous conditioned serum/autologous protein solution (ACS/APS)

\begin{tabular}{|c|c|c|c|c|c|c|c|}
\hline \multirow[t]{2}{*}{ Author and year } & \multirow{2}{*}{$\begin{array}{l}\text { Study } \\
\text { patients }\end{array}$} & \multirow{2}{*}{$\begin{array}{l}\text { Age at presentation } \\
\text { Mean } \\
( \pm \text { SD)/median (range) }\end{array}$} & \multirow{2}{*}{$\begin{array}{l}\text { Follow-up (months) } \\
\text { Mean } \\
( \pm \text { SD)/median (range) }\end{array}$} & \multirow{2}{*}{$\begin{array}{l}\text { Outcome } \\
\text { measure }\end{array}$} & \multirow{2}{*}{$\begin{array}{l}\text { Baseline } \\
\text { Score } \\
\text { Mean }( \pm \text { SD)/median } \\
\text { (range) }\end{array}$} & \multicolumn{2}{|l|}{ Post procedure } \\
\hline & & & & & & $\begin{array}{l}\text { Time point(s) } \\
\text { (weeks/months) }\end{array}$ & $\begin{array}{l}\text { Score } \\
\text { Mean }( \pm \mathrm{SD}) / \text { median (range) }\end{array}$ \\
\hline Kon et al. 2018 [22] & 46 & $57(41-68)$ & $12( \pm \mathrm{NR})$ & WOMAC & $51.2( \pm N R)$ & 12 months & $N R^{a}$ \\
\hline \multirow[t]{3}{*}{ Hang et al. 2017} & \multirow[t]{3}{*}{92} & \multirow[t]{3}{*}{ NR } & \multirow[t]{3}{*}{$12( \pm N R)$} & \multirow[t]{3}{*}{ WOMAC } & Function - 70.3 ( \pm NR) & 12 months & Function - $27.2( \pm N R)$ \\
\hline & & & & & Mobility - $8.6( \pm \mathrm{NR})$ & 12 months & Mobility - $3.1( \pm \mathrm{NR})$ \\
\hline & & & & & Pain - 20.9 ( \pm NR) & 12 months & Pain - 9.0 ( \pm NR) \\
\hline $\begin{array}{l}\text { Shirokova } \\
\text { et al. } 2017 \text { [34] }\end{array}$ & 123 & $59.9( \pm 8.8)$ & $3( \pm N R)$ & WOMAC & $N R^{b}$ & 3 months & $N R^{b}$ \\
\hline Hix et al. 2017 [20] & 10 & $58.8( \pm 9.5)$ & $12( \pm N R)$ & WOMAC & Pain $-12.0( \pm 1.2)$ & 12 months & Pain - $3.3( \pm 2.9)$ \\
\hline $\begin{array}{l}\text { van Drumpt } \\
\text { et al. } 2016 \text { [21] }\end{array}$ & 10 & $57.5( \pm 9.5)$ & $18( \pm 1)$ & WOMAC & $55( \pm N R)$ & 12 months & $20( \pm N R)$ \\
\hline \multirow{4}{*}{$\begin{array}{l}\text { Garcia-Escudero } \\
\text { et al. } 2015 \text { [35] }\end{array}$} & \multirow[t]{4}{*}{118} & \multirow[t]{4}{*}{$59(34-81)$} & \multirow[t]{4}{*}{$24( \pm N R)$} & \multirow[t]{4}{*}{ WOMAC } & Global - $81.6( \pm \mathrm{NR})$ & 12 months & Global - $35.2( \pm \mathrm{NR})$ \\
\hline & & & & & Function - $60.4( \pm \mathrm{NR})$ & 12 months & Function - $29.4( \pm$ NR) \\
\hline & & & & & Stiffness - $3.36( \pm N R)$ & 12 months & Stiffness - $3.3( \pm$ NR) \\
\hline & & & & & Pain - 17.9 ( \pm NR) & 12 months & Pain - $2.5( \pm \mathrm{NR})$ \\
\hline \multirow{4}{*}{$\begin{array}{l}\text { Motaal } \\
\text { et al. } 2014 \text { [37] }\end{array}$} & \multirow[t]{4}{*}{30} & \multirow[t]{4}{*}{$54.21( \pm 5.95)$} & \multirow[t]{4}{*}{$3( \pm N R)$} & \multirow[t]{4}{*}{ WOMAC } & \multirow[t]{4}{*}{ Total - $45.63( \pm 9.99)$} & 1 week & Total - $26.23( \pm 11.50)$ \\
\hline & & & & & & 1 month & Total - $8.0( \pm 7.28)$ \\
\hline & & & & & & 2 months & Total - $7.52( \pm 6.91)$ \\
\hline & & & & & & 3 months & Total - $8.27( \pm 5.45)$ \\
\hline \multirow{12}{*}{$\begin{array}{l}\text { Baltzer } \\
\text { et al. } 2009 \text { [38] }\end{array}$} & \multirow[t]{12}{*}{376} & \multirow[t]{12}{*}{$53.8( \pm 12.2)$} & \multirow[t]{12}{*}{$25( \pm N R)$} & \multirow[t]{12}{*}{ WOMAC } & \multirow[t]{3}{*}{ Global - $5.24( \pm 2.32)$} & 7 weeks & Global - $2.80( \pm 2.30)$ \\
\hline & & & & & & 13 weeks & Global - $2.42( \pm 2.06)$ \\
\hline & & & & & & 26 weeks & Global - $2.42( \pm 2.19)$ \\
\hline & & & & & Pain - $5.18( \pm 2.39)$ & 7 weeks & Pain - $2.71( \pm 2.37)$ \\
\hline & & & & & & 13 weeks & Pain - $2.33( \pm 2.14)$ \\
\hline & & & & & & 26 weeks & Pain $-2.42( \pm 2.25)$ \\
\hline & & & & & Stiffness - $5.59( \pm 2.7)$ & 7 weeks & Stiffness - $3.07( \pm 2.49)$ \\
\hline & & & & & & 13 weeks & Stiffness - $2.80( \pm 2.33)$ \\
\hline & & & & & & 26 weeks & Stiffness - $2.78( \pm 2.45)$ \\
\hline & & & & & Function - $5.21( \pm 2.41)$ & 7 weeks & Function - $2.80( \pm 2.34)$ \\
\hline & & & & & & 13 weeks & Function - $2.40( \pm 2.08)$ \\
\hline & & & & & & 26 weeks & Function - $2.37( \pm 2.21)$ \\
\hline Yang et al. 2008 [39] & 153 & $54( \pm 11)$ & $12( \pm N R)$ & WOMAC & $54.49( \pm 17.6)$ & 3 months & $63.37( \pm 20.6)$ \\
\hline & & & & & & 6 months & $62.90( \pm 23.7)$ \\
\hline & & & & & & 9 months & $61.78( \pm 23.4)$ \\
\hline & & & & & & 12 months & $65.02( \pm 24.1)$ \\
\hline
\end{tabular}

NR

aWOMAC at 12 months reported to be $49 \%$ better but numeric score not provided

${ }^{b}$ WOMAC at 3 months reported to be $28.7 \%$ better but numeric score not provided

components of ACS described include platelet-derived growth factor (PDGF), transforming growth factor-beta (TGF- $\beta$ ), insulin-like growth factor (IGF), and fibroblast growth factor (FGF) amongst others [41, 44, 45]. Hix et al. [20] reported the average concentration of IL-1Ra in APS to be $63,740 \mathrm{pg} / \mathrm{ml}$ whereas Woodell-May et al. [46] noted it to be $30,853 \mathrm{pg} / \mathrm{ml}$. The wide variation in the IL-1Ra levels of ACS and APS reported by the investigators may be due a combination of the production kit and the 24-h incubation period used during the preparation of ACS
[47]. Various anabolic factors, including epidermal growth factor (EGF), vascular endothelial growth factor (VEGF), IGF, TGF- $\beta$ and the anti-inflammatory cytokines soluble tumor necrosis factor receptor type II (sTNF-RII), IL-4 and IL-10, have been noted in APS [46].

\section{Potential mechanisms of action in $O A$}

Broadly, both ACS and APS are injectable solutions enriched in endogenous cytokines which help to restore joint homeostasis preventing degenerative changes in 
Table 5 Reported outcomes - Knee injury and Osteoarthritis Outcome Score (KOOS) and numerical rating score (NRS) following intraarticular injection of autologous protein solution/autologous conditioned serum (ACS/APS)

\begin{tabular}{|c|c|c|c|c|c|c|c|}
\hline \multirow[t]{2}{*}{ Author and year } & \multirow{2}{*}{$\begin{array}{l}\text { Study } \\
\text { patients }\end{array}$} & \multirow{2}{*}{$\begin{array}{l}\text { Age at presentation } \\
\text { Mean } \\
( \pm \mathrm{SD}) / \text { median (range) }\end{array}$} & \multirow{2}{*}{$\begin{array}{l}\text { Follow-up (months) } \\
\text { Mean } \\
( \pm \text { SD)/median (range) }\end{array}$} & \multirow{2}{*}{$\begin{array}{l}\text { Outcome } \\
\text { measure }\end{array}$} & \multirow{2}{*}{$\begin{array}{l}\text { Baseline } \\
\text { Score } \\
\text { Mean ( } \pm \text { SD)/ } \\
\text { median (range) }\end{array}$} & \multicolumn{2}{|l|}{ Post procedure } \\
\hline & & & & & & $\begin{array}{l}\text { Time point(s) (weeks/ } \\
\text { months) }\end{array}$ & $\begin{array}{l}\text { Score } \\
\text { Mean }( \pm \mathrm{SD}) / \\
\text { median (range) }\end{array}$ \\
\hline Kon et al. 2018 [22] & 46 & $57(41-68)$ & $12( \pm N R)$ & KOOS & 39.9 ( $\pm N R)$ & 12 months & $N R$ \\
\hline Hix et al. 2017 [20] & 10 & $58.8( \pm 9.5)$ & 12 ( $\pm \mathrm{NR})$ & KOOS & $36.9( \pm 16.2)$ & 12 months & $79.7( \pm 16.2)$ \\
\hline $\begin{array}{l}\text { Rutgers et al. } 2015 \\
\text { [36] }\end{array}$ & 20 & $50(34-70)$ & $12( \pm N R)$ & KOOS & $49.45( \pm 11.46)$ & 12 months & $51.20( \pm 13.09)$ \\
\hline Hix et al. 2017 [20] & 10 & $58.8( \pm 9.5)$ & $12( \pm \mathrm{NR})$ & NRS & $5.9( \pm 1.9)$ & 12 months & $1.6( \pm 1.6)$ \\
\hline $\begin{array}{l}\text { Garcia-Escudero et al. } \\
2015 \text { [35] }\end{array}$ & 118 & $59(34-81)$ & $24( \pm N R)$ & NRS & $8.10( \pm N R)$ & 12 months & 3.03 ( $\pm N R)$ \\
\hline
\end{tabular}

NR Not reported

cartilage and bone [17, 21, 32, 44, 48]. They offer a nonsurgical intraarticular treatment plan at the molecular level which incorporates the inhibition of IL-1b through the rapid induction of IL-1Ra.

It has been demonstrated that following interaction with medical-grade concentrator beads, the postconditioned serum level of IL-1Ra is significantly elevated in relation to IL-1b $[20,49]$. This relative increase alters the relative ratio of IL-1Ra to IL- $1 \mathrm{~b}$ that is essential to restore homeostasis of joints affected by OA [50].

Whilst IL-1Ra is a major component of these novel agents, their biochemical constituents contain various combinations of PRP and several growth factors present in the a granules of platelets [51]. The aforementioned growth factors, which include TGF- $\beta$, PDGF, VEGF and IGF, have been shown to stimulate chondrocyte proliferation and augment articular cartilage metabolism [44, 52]. Whilst it is perceived that both ACS and APS potentially mitigate the inflammatory cascade, the detailed pathways or mechanisms through which they perform these actions have not been fully described [40, 45, 47, 53].

\section{Limited follow-up and natural history of $O A$}

There is paucity of robust data in the literature to evaluate the long-term benefits of autologous cell-free serum preparations in the management of knee OA. This is vital for a condition such as OA with multifactorial aetiology and treatments that influence the prognosis. Zarringam [23] et al. attempted to answer this question in the cohort of patients who had participated in a study [39] a decade earlier. It must be observed that in this study the composition of patient groups that reported the outcomes were based on certain assumptions thereby limiting the interpretation of the results.

\section{Imaging features}

In their study Kon et al. [22] analysed the effect of the intraarticular administration of APS on the size of bone- marrow lesion and osteophytes over a 12-month period using the Magnetic resonance imaging OsteoArthritis Knee Score (MOAKS). They observed that in the central zone of the lateral femoral condyle these lesions significantly improved in the study population. However, these changes were not statistically significant. Of note, neither the study protocol as listed on ClinicalTrials.gov [54], nor the 'Methods' section, specify how the magnetic resonance imaging (MRI) images were planned to be analysed. Nonetheless, this an area for further investigation as it has been demonstrated that bone-marrow lesions contribute to significant symptoms in patients with knee OA $[55,56]$.

\section{Outcome measures and clinical factors}

The visual analogue scale (VAS) and Western Ontario and McMaster Universities osteoarthritis instrument (WOMAC) are the most commonly used outcome measures in the current literature (Tables 3 and 4). Some studies have used the Knee Injury and Osteoarthritis Outcome Score (KOOS) and the numeric rating scale (NRS) to report the results of injection of ACS/APS (Table 5). However, there is lack of consensus on the outcome measures to report the results of this procedure.

Some authors [57] have hypothesised that the preparation and administration of autologous cell-free serum preparations in patients with a raised C-reactive protein (CRP) can yield suboptimal results due to the elevated levels of pro-inflammatory cytokines (IL-1, TNF) in the same blood.

There is considerable variation in the technique of intraarticular administration of ACS/APS as highlighted earlier. Some authors [21, 23, 39] recommend aspiration of the knee prior to injection to reduce the risk of drug dilution. However, this step is not reported consistently in the other studies [31, 35, 37]. Synovial fluid analysis is performed as a diagnostic investigation for knee conditions including crystal arthropathy [58]. It is interesting 
to note that no study has analysed the aspirate fluid to determine the suitability of patients to this treatment procedure.

BMI has been reported to be an independent risk factor for the development [59] and progression of symptoms [60] in patients with knee OA. Limb malalignment in the coronal/sagittal plane can contribute to knee-joint degeneration and wear [61, 62]. However, the current studies lack uniform data on these vital factors which predict the outcome of any intervention in this set of patients (Table 2). Another confounding factor in the studies investigating the effect of ACS/APS is the inconsistent criteria for the use of type and dose of adjunctive analgesics in the study patients. This has to be considered whilst interpreting the results of the studies.

It must be mentioned that the largest series (over 1000 patients) on the topic is available in the German literature [63] and is not included in the current review. Nonetheless, in this study no significant adverse outcomes were reported.

\section{Cost of ACS/APS treatment regimen}

No study in the literature has directly compared the cost of autologous cell-free serum preparations in the management of knee OA with other treatment options. However, it must be mentioned that the cost implications of products like ACS/APS can be inferred from various commercial sources. In their study, Barreto et al. [31] stated that ACS was a relatively cost-effective treatment compared to other injection treatments like PRP, stem cells or surgical procedures like total knee replacement. Orthokin (Orthogen AG, Dusseldorf, Germany) has a stated cost of $€ 150-750$ for ACS therapy $(\approx$ US\$170-855) [64], whilst the nSTRIDE ${ }^{\circ}$ APS kit is quoted at $£ 770(\approx$ US\$1016) excluding the adjunctive equipment [65]. Synvisc ${ }^{\circ}$ (Sanofi, Paris, France), a representative HA injection costs $£ 68.33$ ( $\approx$ US\$87.85) per injection [66]. By comparison, Kenalog (Bristol-Myers Squibb, New York, NY, USA) containing triamcinolone acetonide has a British National Formulary indicative price of $£ 1.49(\approx$ US\$1.92) per 40-mg vial [67]. The cost of PRP therapy varies from $£ 425-1200$ ( $\approx$ US\$545-1540) [68] whereas the guide price for hospital-based autologous stem-cell therapy for knee OA may be higher at $£ 7500(\approx$ US\$9626) [69].

\section{Summary}

The overall quality of evidence supporting ACS/APS use for OA of the knee is poor, with considerable heterogeneity between trials and a paucity of large, well-conducted randomised controlled trials. There is some evidence that ACS/APS is effective in the short-to-medium term (3-24 months) control of pain from OA as well as improving range of movement and function. The rate of serious complications is low, and there is no evidence that future surgery is compromised by prior ACS/APS injection. There have been no formal health economic analyses of ACS/APS compared to more established intraarticular therapies.

\section{Conclusions}

Limited data from the current studies would suggest that intraarticular administration of autologous cell-free serum preparations, such as ACS/APS, in patients with knee OA may improve pain and function with limited morbidity. Given the heterogenous data in the literature it may be useful to develop blood or synovial tests that may predict an efficacious result from ACS/APS. However, high-quality clinical trials with stratified patient cohorts, longer follow-up duration and robust reporting of outcome measures are essential to improve the current understanding of the indications and clinical effectiveness of these products.

\section{Abbreviations \\ APS: Autologous protein solution; ACS: Autologous conditioned serum; OA: Osteoarthritis; IAC: Intraarticular corticosteroid; HA: Hyaluronic acid; IL- 1: Interleukin-1; IL-1Ra: Interleukin-1-receptor antagonist; IL-6: Interleukin-6; TNF-a: Tumor necrosis factor-alpha; MCP: Macrophage chemotactic protein; MIG: Monokine induced by interferon; OSM: Oncostatin M; MMPs: Matrix metalloproteinases; PRP: Platelet-rich plasma; MSCs: Mesenchymal stem/ stromal cells; SD: Standard deviation; NR: Not reported; K-L: Kellgren- Lawrence; ACR: American College of Rheumatology criteria; VAS: Visual analogue scale; KOOS: Knee injury and Osteoarthritis Outcome Score; WOMAC: Western Ontario and McMaster Universities osteoarthritis instrument; SF-8: Short-Form 8 health-related quality of life; K-M: Kaplan- Meier method; CGI-S: Clinical Global Impression of Severity; PGI-S: Patient Global Impression of Severity; XSMFA: D-Extra Short Musculoskeletal Functional Assessment; KSCRS: Knee Society Clinical Rating Scale; BMI: Body mass index; NRS: Numeric rating scale; CRP: C-reactive protein; MOAKS: Magnetic resonance imaging OsteoArthritis Knee Score}

\section{Acknowledgements \\ None}

Authors' contributions

All the authors contributed towards the literature search, critical review, data analysis and manuscript preparation. The author(s) read and approved the final manuscript.

\section{Authors' information}

What is known about the subject: autologous cell-free serum preparations such as ACS/APS are nonoperative treatment options in patients with knee osteoarthritis. Current studies have reported improvement in pain and function of patients with knee osteoarthritis.

What this study adds to existing knowledge: this review paper collates the available evidence regarding this procedure. It demonstrates that: (1) autologous cell-free serum preparations, such as ACS/APS, may improve pain and function of patients with knee osteoarthritis with limited morbidity and complications; (2) only limited data is available describing the long-term clinical effectiveness of this procedure; and (3) considerable variation exists in the outcome measures used to report the results. It highlights the need for pragmatic, prospective studies with long-term follow-up and robust reporting of results to further our understanding of this potentially useful treatment modality. 


\section{Availability of data and materials}

Presented in the manuscript

\section{Competing interests}

The authors declare that they have no competing interests.

Received: 15 September 2019 Accepted: 27 February 2020

Published online: 23 March 2020

\section{References}

1. Kiadaliri AA, Lohmander LS, Moradi-Lakeh M, Petersson IF, Englund M (2018) High and rising burden of hip and knee osteoarthritis in the Nordic region, 1990-2015: findings from the Global Burden of Disease Study 2015. Acta Orthop 89(2):177-183

2. Liu Q, Niu J, Li H, Ke Y, Li R et al (2017) Knee symptomatic osteoarthritis, walking disability, NSAIDs use and all-cause mortality: population-based Wuchuan osteoarthritis study. Sci Rep 7(1):3309

3. Yates AJJ, McGrory BJ, Starz TW, Vincent KR, McCardel B et al (2014) AAOS appropriate use criteria: optimizing the non-arthroplasty management of osteoarthritis of the knee. J Am Acad Orthop Surg 22(4):261-267

4. Abbott J, Wilson R, Pinto D, Chapple CM, Wright AA et al (2019) Incremental clinical effectiveness and cost effectiveness of providing supervised physiotherapy in addition to usual medical care in patients with osteoarthritis of the hip or knee: 2-year results of the MOA randomised controlled trial. Osteoarthr Cartil 27(3):424-434

5. Messier SP, Resnik AE, Beavers DP, Mihalko SL, Miller GD et al (2018) Intentional weight loss in overweight and obese patients with knee osteoarthritis: is more better? Arthritis Care Res 70(11):1569-1575

6. Kyung H-S (2016) High tibial osteotomy for medial knee osteoarthritis. Knee Surg Relat Res 28(4):253-254

7. Passias PG, Bono OJ, Bono JV (2018) Total knee arthroplasty in patients of advanced age: a look at outcomes and complications. J Knee Surg 33(1):1-7

8. Healy WL, Della Valle CJ, lorio R, Berend KR, Cushner FD et al (2013) Complications of total knee arthroplasty: standardized list and definitions of the Knee Society. Clin Orthop Relat Res 471(1):215-220

9. Barker KL, Hannink E, Pemberton S, Jenkins C (2018) Knee arthroplasty patients predicted versus actual recovery: what are their expectations about time of recovery after surgery and how long before they can do the tasks they want to do? Arch Phys Med Rehabil 99(11):2230-2237

10. Koenig KM, Ong KL, Lau EC, Vail TP, Berry DJ et al (2016) The use of hyaluronic acid and corticosteroid injections among Medicare patients with knee osteoarthritis. J Arthroplast 31(2):351-355

11. Syed HM, Green L, Bianski B, Jobe CM, Wongworawat MD (2011) Bupivacaine and triamcinolone may be toxic to human chondrocytes: a pilot study. Clin Orthop Relat Res 469(10):2941-2947

12. Wehling P, Evans C, Wehling J, Maixner W (2017) Effectiveness of intraarticular therapies in osteoarthritis: a literature review. Ther Adv Musculoskel Dis 9(8):183-196

13. Altman R, Hackel J, Niazi F, Shaw P, Nicholls M (2018) Efficacy and safety of repeated courses of hyaluronic acid injections for knee osteoarthritis: a systematic review. Semin Arthritis Rheum 48(2):168-175

14. Jevsevar DS (2013) Treatment of osteoarthritis of the knee, 2nd edition: evidence-based quideline. J Am Acad Orthop Surg 21(9):571-576

15. Wu Y-T, Hsu K-C, Li T-Y, Chang C-K, Chen L-C (2018) Effects of platelet-rich plasma on pain and muscle strength in patients with knee osteoarthritis. Am J Phys Med Rehabil 97(4):248-254

16. lijima H, Isho T, Kuroki H, Takahashi M, Aoyama T (2018) Effectiveness of mesenchymal stem cells for treating patients with knee osteoarthritis: a meta-analysis toward the establishment of effective regenerative rehabilitation. NPJ Regen Med 3(1):1-13

17. Vitale ND, Vandenbulcke F, Chisari E, lacono F, Lovato L et al (2019) Innovative regenerative medicine in the management of knee OA: the role of autologous protein solution. J Clin Orthop Trauma 10(1):49-52

18. O'Shaughnessey K, Matuska A, Hoeppner J, Farr J, Klaassen M et al (2014) Autologous protein solution prepared from the blood of osteoarthritic patients contains an enhanced profile of anti-inflammatory cytokines and anabolic growth factors. J Orthop Res 32(10):1349-1355

19. Frisbie DD, Kawcak CE, Werpy NM, Park RD, Mcllwraith CW (2007) Clinical, biochemical, and histologic effects of intra-articular administration of autologous conditioned serum in horses with experimentally induced osteoarthritis. Am J Vet Res 68(3):290-296
20. Hix JK, Klaassen M, Foreman R, Cullen E, Toler K, King W, Woodell-May J (2017) An autologous anti-inflammatory protein solution yielded a favorable safety profile and significant pain relief in an open-label pilot study of patients with osteoarthritis. Biores Open Access 6(1):151-158

21. van Drumpt RAM, van der Weegen W, King W, Toler K, Macenski MM (2016) Safety and treatment effectiveness of a single autologous protein solution injection in patients with knee osteoarthritis. Biores Open Access 5(1):261-268

22. Kon EE, Engebretsen L, Verdonk P, Nehrer S, Filardo G (2018) Clinical outcomes of knee osteoarthritis treated with an autologous protein solution injection: a 1-year pilot double-blinded randomized controlled trial. Am J Sports Med 46(1):171-180

23. Zarringam DB, Bekkers JEJ, Saris DBF (2018) Long-term effect of injection treatment for osteoarthritis in the knee by Orthokin autologous conditioned serum. Cartilage 9(2):140-145

24. Tassara MDP, De Ponti A, Barzizza L, Zambelli M, Parisi C, Milani R, Santoleri L (2018) Autologous conditioned serum (ACS) for intraarticular treatment in osteoarthritis: retrospective report of 28 cases. Transfus Apher Sci 57(4):573-577

25. Moser C, Baltzer AW (2009) Treatment of knee osteoarthritis with autologous conditioned serum (ACS): a prospective, randomized, placebocontrolled, patient- and observer-blind, parallel-design trial. Therapeutic study, level I (randomized controlled trial-RCT, ISRCTN: 71311752). J Bone Joint Surg Proc 91-B(Suppl_l):157-115a

26. Rutgers M, Saris DB, Dhert WJ, Creemers LB (2010) Cytokine profile of autologous conditioned serum for treatment of osteoarthritis, in vitro effects on cartilage metabolism and intra-articular levels after injection. Arthritis Res Ther 12(3):R114

27. Bertone AL, Ishihara A, Zekas LJ, Wellman ML, Lewis KB et al (2014) Evaluation of a single intra-articular injection of autologous protein solution for treatment of osteoarthritis in horses. Am J Vet Res 75(2):141-151

28. Multidisciplinary European Database. Available at: http://www.opengrey.eu/ search/. Accessed 24 Nov 2019.

29. OpenDOAR: Directory of Open Access Repositories. Available at: http://v2. sherpa.ac.uk/opendoar/. Accessed 24 Nov 2019.

30. Hang DW (2015) Efficacy study of the treatment of osteoarthritis-induced knee pain with autologous conditioned serum: A comparative, prospective and randomized study. Osteoporos Int 26(1):56

31. Barreto A, Braun TR (2017) A new treatment for knee osteoarthritis: clinical evidence for the efficacy of Arthrokinex ${ }^{\mathrm{TM}}$ autologous conditioned serum. J Orthop 14(1):4-9

32. Barreto A, Braun TR (2017) A new treatment for knee osteoarthritis: clinical evidence for the efficacy of ArthrokinexTM autologous conditioned serum. J Orthop 14(1):4-9

33. Hang D (2017) Treatment of osteoarthritis knee with autologous conditioned serum: Comparative, prospective study. Osteoporos Int 28(1):160

34. Shirokova KN, Noskov S, Shirokova L (2017) Comparison of clinical efficacy of platelet-rich plasma and autologous conditioned serum treatment in patients with osteoarthritis of the knee. Osteoarthritis Cartilage 25(1):S438

35. García-Escudero JB, Miguel Hernández Trillos P (2015) Treatment of osteoarthritis of the knee with a combination of autologous conditioned serum and physiotherapy: a two-year observational study. PLoS One 10(12): e0145551

36. Rutgers M, Creemers LB, Auw Yang KG, Raijmakers NJH, Dhert WJA et al (2015) Osteoarthritis treatment using autologous conditioned serum after placebo. Acta Orthop 86(1):114-118

37. Motaal FE, Elganzouty AM, Fathalla MM, Abdulkareem O (2014) Low-dose intra-articular autologous conditioned serum in treatment of primary knee osteoarthritis. Egypt Rheumatol Rehabil 41(3):98

38. Baltzer AWA, Moser C, Jansen SA, Krauspe R (2009) Autologous conditioned serum (Orthokine) is an effective treatment for knee osteoarthritis. Osteoarthr Cartil 17(2):152-160

39. Yang KR, Raijmakers NJH, van Arkel ERA, Caron JJ, Rijk PC, Willems WJ, Zijl JAC, Verbout AJ, Dhert WJA, Saris DBF (2008) Autologous interleukin-1 receptor antagonist improves function and symptoms in osteoarthritis when compared to placebo in a prospective randomized controlled trial. Osteoarthr Cartil 16(4):498-505

40. Richards MM, Maxwell JS, Weng L, Angelos MG, Golzarian J (2016) Intraarticular treatment of knee osteoarthritis: from anti-inflammatories to products of regenerative medicine. Phys Sportsmed 44(2):101-108

41. Evans $\mathrm{CH}$, Chevalier $X$, Wehling $P$ (2016) Autologous conditioned serum. Phys Med Rehabil Clin 27(4):893-908 
42. nSTRIDE APS versus hyaluronic acid for knee osteoarthritis. Available at: https://clinicaltrials.gov/ct2/show/NCT03182374. Accessed 1 Feb 2020.

43. Biomet Z (2018) nSTRIDE ${ }^{\circledR}$ autologous protein solution kit. In: ZimmerBiomet

44. Wehling P, Moser C, Frisbie D, Mcllwraith CW, Kawcak CE et al (2007) Autologous conditioned serum in the treatment of orthopedic diseases. BioDrugs 21(5):323-332

45. Wehling P, Moser C, Maixner W (2016) How does surgery compare with advanced intra-articular therapies in knee osteoarthritis: current thoughts. Ther Adv Musculoskelet Dis 8(3):72-85

46. Woodell-May J, Matuska A, Oyster M, Welch Z, O'Shaughnessey K et al (2011) Autologous protein solution inhibits MMP-13 production by IL-1 $\beta$ and TNFastimulated human articular chondrocytes. J Orthop Res 29(9):1320-1326

47. Linardi RL, Dodson ME, Moss KL, King WJ, Ortved KF (2019) The Effect of Autologous Protein Solution on the Inflammatory Cascade in Stimulated Equine Chondrocytes. Front Vet Sci 6:64

48. Fox BA, Stephens MM (2010) Treatment of knee osteoarthritis with Orthokine-derived autologous conditioned serum. Expert Rev Clin Immunol 6(3):335-345

49. Barreto A, Braun TR (2016) A method to induce interleukin-1 receptor antagonist protein from autologous whole blood. Cytokine 81(1):137-141

50. Chevalier X, Giraudeau B, Conrozier T, Marliere J, Kiefer P et al (2005) Safety study of intraarticular injection of interleukin 1 receptor antagonist in patients with painful knee osteoarthritis: a multicenter study. J Rheumatol 32(7):1317-1323

51. Ulrich-Vinther M, Maloney MD, Schwarz EM, Rosier R, O'Keefe RJ (2003) Articular cartilage biology. J Am Acad Orthop Surg 11(6):421-430

52. Frizziero A, Giannotti E, Oliva F, Masiero S, Maffulli N (2012) Autologous conditioned serum for the treatment of osteoarthritis and other possible applications in musculoskeletal disorders. Br Med Bull 105(1):169-184

53. Frisbie DD (2015) Autologous-conditioned serum: evidence for use in the knee. J Knee Surg 28(1):63-66

54. APSS-33-00: a multicenter, pilot study of Autologous Protein Solution (APS) in Knee Osteoarthritis (OA) (PROGRESS II). Available at:https://clinicaltrials. gov/ct2/show/NCT02138890. Accessed 1 Feb 2020

55. Collins JA, Beutel BG, Strauss E, Youm T, Jazrawi L (2016) Bone marrow edema: chronic bone marrow lesions of the knee and the association with osteoarthritis. Bulletin Hosp Jt Dis 74(1):24

56. Roemer FW, Neogi T, Nevitt MC, Felson DT, Zhu Y et al (2010) Subchondral bone marrow lesions are highly associated with, and predict subchondral bone attrition longitudinally: the MOST study. Osteoarthr Cartil 18(1):47-53

57. Crnogaca K, Bicanic G, Delimar D (2016) Elevated CRP level could herald less efficient autologous conditioned serum (ACS) treatment. Med Hypotheses 86:135-137

58. Sholter D, Russell A, Calderwood SB (2017) Synovial fluid analysis. UpToDate, Waltham Accessed 16 Mar 2017

59. Zheng H, Chen C (2015) Body mass index and risk of knee osteoarthritis: systematic review and meta-analysis of prospective studies. BMJ Open 5(12): e007568

60. Weiss $E$ (2014) Knee osteoarthritis, body mass index and pain: data from the Osteoarthritis Initiative. Rheumatology 53(11):2095-2099

61. Felson DT, Niu J, Gross KD, Englund M, Sharma L et al (2013) Valgus malalignment is a risk factor for lateral knee osteoarthritis incidence and progression: findings from the Multicenter Osteoarthritis Study and the Osteoarthritis Initiative. Arthritis Rheum 65(2):355-362

62. Hayashi D, Englund M, Roemer FW, Niu J, Sharma L et al (2012) Knee malalignment is associated with an increased risk for incident and enlarging bone marrow lesions in the more loaded compartments: the MOST study. Osteoarthr Cartil 20(11):1227-1233

63. Baltzer A, Drever R, Granrath M, Godde G, Klein W, Wehling P (2003) Intraartikuläre Therapie der Gonarthrose mit autologem Interleukin-1 Rezeptor Antagonisten (IL-1Ra). Deutsche Zeitschrift für Sportmedizin. 54(6): 209-11

64. Orthogen Laboratory Services: Doctor's Questions and our Answers. Available at: https://orthogen.com/orthokin/en/faculty/questions/\#faq20. Accessed 24 Nov 2019.

65. Zimmer Biomet: nSTRIDE Price. Email correspondence. Available at: https:// www.zimmerbiomet.com. Accessed 22 Jan 2019.
66. Monthly Index of Medical Specialities: Synvisc. Available at: https://www. mims.co.uk/drugs/musculoskeletal-disorders/topical-analgesics-localinjections/synvisc. Accessed 24 Nov 2019.

67. British National Formulary: Triamcinolone Acetonide. Available at: https:// bnf.nice.org.uk/medicinal-forms/triamcinolone-acetonide.html. Accessed 24 Nov 2019.

68. Platelet Rich Plasma (PRP) Injections - Outpatient Pricing. Available at: https://www.royalfreeprivatepatients.com/treatments/platelet-rich-plasmainjections/. Accessed 24 Nov 2019.

69. Knee Autologous Stem Cell Therapy (W3110). Available at: https://www. highgatehospital.co.uk/patient/payment/self-funding-patients/guide-prices/. Accessed 24 Nov 2019.

\section{Publisher's Note}

Springer Nature remains neutral with regard to jurisdictional claims in published maps and institutional affiliations.
Ready to submit your research? Choose BMC and benefit from:

- fast, convenient online submission

- thorough peer review by experienced researchers in your field

- rapid publication on acceptance

- support for research data, including large and complex data types

- gold Open Access which fosters wider collaboration and increased citations

- maximum visibility for your research: over $100 \mathrm{M}$ website views per year

At $\mathrm{BMC}$, research is always in progress.

Learn more biomedcentral.com/submissions 